\title{
Global path planning of Multi-robot Fish based on adaptive ant colony
}

\section{algorithmin dynamic environment}

\author{
Cai Wen1, Yansong Deng* \\ Institute of Electrical and Information Engineering, Southwest University for Nationalities of \\ China,Chengdu, Sichuan, 610041, P.R.China \\ *Corresponding author. Email:342547288@qq.com
}

Keywords:adaptiveant colony algorithm, path planning, multi-robot fish

\begin{abstract}
Based on adaptive ant colony algorithm, this paper provides a global path planning for multi-robot fishin dynamic environment, so as to achieve better coordination between the robot fish by means of improving their path planning. The adaptive ant colony algorithmin combination with dynamic self-adapting adjust pheromone, can avoid local minimum and have good global search ability. It can effectively expand the search space and quickly findan optimal path to reach the destination.In the unknown obstacle environment which is rasterized, target nodes are generated via obstacle avoidance constraint and adjacent grid transition probabilityto complete the selection of each path.By many experiments in matlab,the results show that the method can let multi-robot fish avoid obstacles to reach the destination effectively in dynamic obstacle environment.
\end{abstract}

\section{Introduction.}

With the increasing development of biomimetic fish in water transportation, military reconnaissance and other more complex water environment, multi-robot fish are needed to adapt to environmental constraintsthrough cooperation to reach destination. And global path planning is the basic problem for multi-robot fishto accomplish tasks.At present, there are many methods for global path planning of the robot. Such as artificial potential field[1], neural network method[2] and so on. But artificial potential field method is easy to produce the local optimum and the deadlock problem. As for neural network method, when there are more obstacles in the environment, the threshold of neurons needs to constantly change with time. To better solve the issues, this paper applies the adaptive ant colony algorithm to achieve the path planning of multi-robot fish in dynamic obstacles environment. This algorithm use adaptive adjustment method to update pheromone, which can keep the algorithm has positive and negative feedback mechanism, so as to find the optimal solution while keeping the search scope, avoiding prematurely converge to unsatisfactory results. The Characteristics ofheuristic search and distributed computing can make optimal solution of global path is effectively obtained.

\section{Environment modeling}

Environment modeling is based on grid identification method. The starting point is $\mathrm{S}$ and the target point is T.Setting obstacles in random positionthrough the establishment of random matrix.As for grid, number from left to right, from top to bottom. The working space of the robot is set up by the $\mathrm{M}$ line $\mathrm{N}$ column grid.Arbitrary grid number $\mathrm{R}$ corresponds grid row and column number are $\mathrm{x}$, $\mathrm{y}$. The relationship is $\mathrm{X}=\mathrm{R} \% \mathrm{~N}+1, \mathrm{Y}=\mathrm{R} / \mathrm{N}+1$. Take the obstacle map to a two dimensional array matrix 
$\operatorname{map}(m, n)$. if $(m, n)$ on a barrier, $\operatorname{map}(m, n)=1$, otherwise, $\operatorname{map}(m, n)=0$.

\section{The mathematical model ofBasic ant colony algorithm}

Studies have found that each ant leaves behind a chemical substance that is an element of pheromone on its path.In a certain range the other ants can detect the substance andtend to move toward a higher concentration of pheromone.The higher concentration of pheromone on a certain path, the greater probability that the ant will choose this path.Ants through the exchange of pheromone, and ultimately choose the shortest path to search for food. In this algorithm, ants according to pheromone concentration on each path, using pseudo random probability rule to choose the next step to move the position. The probability that kant $(\mathrm{k}=1,2,3 \ldots, \mathrm{m})$ will move from the grid $i$ to the $j$ at the $t$ time is defined $\operatorname{asp}_{\mathrm{ij}}^{\mathrm{k}}(\mathrm{t})$,as follows:

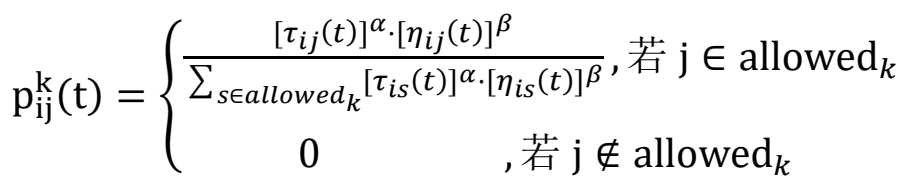

$\tau_{i j}(t)$ is pheromone concentration of the path $\langle\mathrm{i}, \mathrm{j}\rangle$ at time t. $\eta_{i j}(t)$ is heuristic function of $\mathrm{i}$ to j. $\alpha$ is heuristic factor,reflecting the relative importance of pheromone. $\alpha$ is larger, the ant is more inclined to choose the path of other ants. $\beta$ is expectation heuristic factor, reflectingthe importance of the heuristic function $\eta_{i j}(t) . \beta$ is larger, the more likely to choose the nearest path point. Since ant colony algorithm has memory function, the allowedk $=\{\mathrm{V}$ - tabuk $\}$ is that kant can choose the next grid number. $\mathrm{V}$ is a collection of $\mathrm{N}$ nodes.tabuk is taboo list, which is used to record the current position that thekant has already passed by.Thereinto, $\eta_{i j}$ is related to the distance $\eta_{i j}=\frac{1}{d_{i j}}$. dij is the distance between two adjacent nodes $\mathrm{i}$ and $\mathrm{j}$. As for $\mathrm{K}$ ant, the dij is smaller, the $\eta_{i j}(t)$ is larger, the $\mathrm{p}_{\mathrm{ij}}^{\mathrm{k}}(\mathrm{t})$ becoming large. The state transition probability is more close to the greedy rule

\section{Adaptive ant colony algorithm}

After each ant walk one step or complete the traversal of all $\mathrm{N}$ nodes, need to update the residual information. if $\tau_{i j}(t)$ is pheromone concentration of the path $\langle i, j>$ at time $t$, the $t+1$ time on the path of the pheromone concentration is:

$$
\begin{gathered}
\tau_{i j}(t+1)=(1-\rho) \tau_{i j}(t)+\sum_{k=1}^{m} \Delta \tau_{i j}^{k}(t) \quad \text { (2) } \\
\Delta \tau_{i, j}^{k}(t, t+n)=\left\{\begin{array}{c}
\frac{Q}{L_{k}}, \text { If the ants in this cycle through the path }<i, j> \\
0, \text { else }
\end{array}\right.
\end{gathered}
$$

$\rho$ is pheromone evaporation factor, $\rho \in[0,1)$ and the $1-\rho$ is pheromone concentration residue factor,reflecting relative importance of residual pheromone. $\Delta \tau_{i j}^{k}(t)$ is pheromoneincrementof the $\mathrm{K}$ ant on the path $\langle\mathrm{i}, \mathrm{j}\rangle$ from $\mathrm{t}$ time to $\mathrm{t}+1$ time.In initial moment, $\Delta \tau_{i j}(0)=0, \mathrm{Q}$ is the total amount of pheromone released by ants. $\mathrm{L}_{\mathrm{k}}$ is the path length of the $\mathrm{K}$ ant in this cycle.The entire pheromone update is designed to inject additional information on the shortest route.In other words, only the pheromone that belongs to the shortest path can be enhanced.This is a positive feedback process. When the robot passes through a node, the corresponding information is recorded. 
But each candidate solution is constantly adjusting its structure according to the accumulated information. The more ants on the path, the greater the pheromone, the easier it is to choose the path,easily causing to local optimum. Aimed at this problem, this paper discuss an ant colony algorithm based on adaptive pheromone evaporation factor $\rho . \rho$ has a direct impact on the global search capability and convergence rate of ant colony algorithm. In order to improve the global search ability of the basic ant colony algorithm and to improve its speed,making the following changes: (1)After the end of each cycle, the optimal solution is kept. (2) To adaptive change $\rho$. if $\rho$ is too large, the information on the path that has not been searched is reduced to close to 0,reducing global search capability. ifreducing $\rho$ will reduce the convergence rate of the algorithm. So setting $\rho(\mathrm{t} 0)=1$. When the optimal solution is not obviously improved in the $\mathrm{N}$ sub cycle. P reduced to

$$
\rho(\mathrm{t})=\left\{\begin{array}{cl}
0.95 \rho(t-1), & (\mathrm{x}, \mathrm{y}) \text { on a barrier } \\
\rho_{\min }, & \text { else }
\end{array}\right.
$$

$\rho_{\text {min }}$ is the minimum of $\rho$, which can prevent $\rho$ so small that convergence rate of algorithmis reduced.

\section{Algorithm flowchart of this paper}

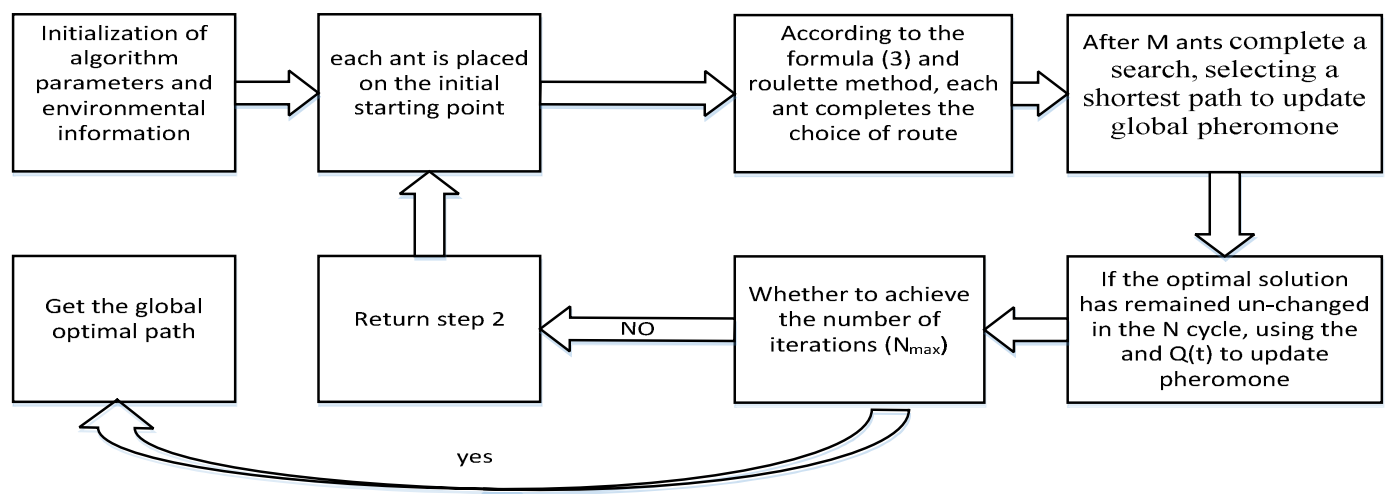

Fig.1 The operation of the adaptive ant colony algorithm

\section{Experiments and analysis.}

In order to verify the theory's effectiveness and correctness, the author does the simulation experience in MATLAB.The robot's working environment is divided into 30*30 grid.Each small square lattice pixel length is set to 1 . Number of ants is 50. In a randomly generated obstacle environment,four experiments were carried out, then to compare. The first two experiments are based on adaptive ant colony algorithm, and others are based on basic ant colony algorithm $(\beta=5$, $\alpha=1, \mathrm{Nmax}=100, \rho_{\min }=0.1$ ) 


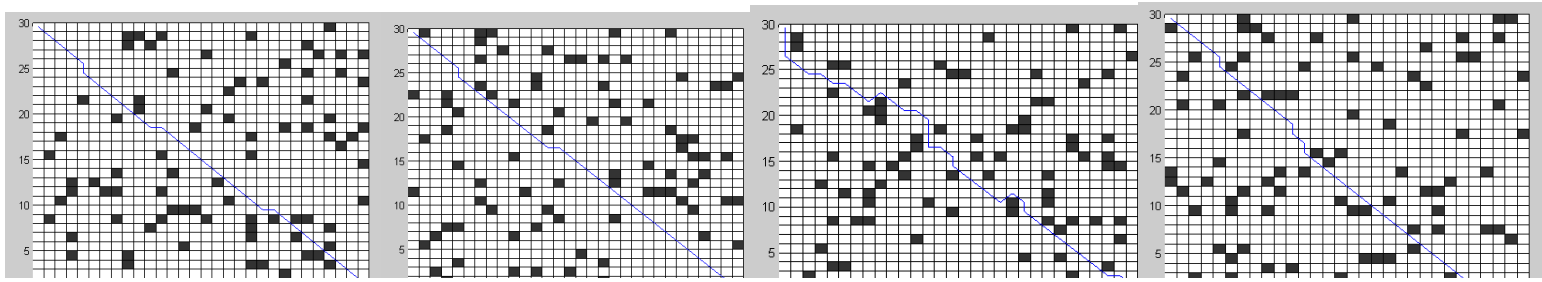

(a) environment 1

(b) environment 2

(a) environment 3

(b) environment 4

Fig.2Optimal path trajectorybased on adaptive algorithm

Fig.3Optimal path trajectorybased on basicalgorithm

From Fig.2,this is the convergence path of the robot's final moments.Under the two different obstacles environment, the robot all can find a way to avoid obstacles to reach the destination from the starting point.Path trajectory is relativelysmooth.From Fig.3,although the convergence path of the robot's final moments also can avoid obstacle to reach the destination,path trajectory is more curve than Fig.2 Global planning performance is worse than the former.

Table1Performance comparison of algorithms

\begin{tabular}{|r|r|r|r|r|}
\hline Environment & environment & environment & environment & environment \\
\hline Path length & 43.3533 & 43.0122 & 48.1838 & 44.7696 \\
\hline Time consumir & 65.266764 & 64.467463 & 95.073804 & 84.706815 \\
\hline
\end{tabular}

From the Table1,adaptive ant colony algorithmcan avoid the search into local optimal,and reduce the robot's walking time. The method can effectively solve the problem of path planning for the multi-robot fish in different obstacles environment.

\section{Conclusion.}

This paper mainly introduces the global path planning based on adaptive ant colony algorithm in obstacles environment. The combination of pheromone adaptive adjustment and heuristic function make the algorithm has a more comprehensive search ability and search speed.With the method's strong obstacle avoidance and path planning ability, the multi-robot fish can batter cooperate and communicate in the complex water environment.

\section{Acknowledgement}

This work was supported by the SWUT students' innovative training program (No. 201610656038)

\section{Reference}

[1]Xiaohao Xu, Chenggong Li, Xiong Li. Modified route planning based on artificial potential field algorithm [J]. Journal of traffic and Transportation Engineering,2009,06:64-68.

[2]Lei Huang. Research on path planning of mobile robot based on Neural Network [D]. Wuhan University of Technology,2008.

[3]Lila Zhu. Research on multi robot formation based on swarm intelligence algorithm [J]. Computer measurement and control ,2011,04:990-992+997.

[4]Bingmei Chen, Xiaoping Fan, Zhiming Zhou, Xuerong Li, Matlab ant colony simulation for 
solving traveling salesman problem [J]. Computer measurement and control,2011,04:990-992+997.

[5]Enxiu Shi, Minmin Chen, Jun Li, Yumei Huang, Research on global path planning for mobile robot based on ant colony algorithm [J]. Chinese Journal of agricultural machinery,2014,06:53-57.

[6]Rui Chu, UAV route planning based on ant colony algorithm [D]. Northwestern Polytechnical University,2006.

[7]Xuefeng Yang, Research on ant colony algorithm for solving TSP problem [D]. Jilin University,2010. 